\title{
Novel UV-activated biofunctionalization of up-converting nanocrystals for detection of proteins
}

\author{
Małgorzata Misiak ${ }^{1,2}$ (D) Maciej Gawłowski ${ }^{1} \cdot$ Agnieszka Kowalczyk $^{1} \cdot$ Michał Skowicki $^{1} \cdot$ Katarzyna Prorok $^{2}$. \\ Tomasz Lipiński ${ }^{1}$
}

Received: 20 October 2020 / Accepted: 8 April 2021 / Published online: 17 April 2021

(c) The Author(s) 2021

\begin{abstract}
Lanthanide doped nanocrystals capable to emit higher energy photons under excitation with lower energy radiation are promising for a broad range of applications including biodetection, biosensing, and bioimaging. However, the adaptation of these nanoparticles to the biological environment that requires good water-solubility, stability and ease of further functionalization still remains a challenge. The application of nanoparticles for biodetection or in various assays encountered many difficulties arising mainly from the strong tendency of nanoparticles for aggregation or nonspecific binding. Here we present a new method to obtain soluble and stable in water-based buffers $\mathrm{NaYF}_{4}: \mathrm{Yb}^{3+} \mathrm{Er}^{3+}$ nanocrystals with modified surface ready for further conjugation with biomolecules. In the presented approach polyvinylpyrrolidone/vinyl alcohol copolymer (PVP/ VA) with photo-activatable linker (N-5-Azido-2-nitrobenzoyl group-ANB-NOS) was used for initial coating due to its high non-covalent affinity to nanoparticles surface. Subsequent coating with aminated dextran by ultraviolet light activation of ANB-NOS was carried out. This step has a significant impact on nanocrystals stability in the physiological buffer as well as on protein conjugation. Conjugation of biomolecules was possible by employing another photo-linker-sulfosuccinimidyl 4,4'-azipentanoate (sulfo-SDA). Bacterial Protein G has been selected to create a universal immune-imaging agent due to its ability to bind $\mathrm{Fc}$ fragment of most mammalian immunoglobulins. Moreover, the direct attachment of antibodies to nanoparticles was also examined. The activity of biofunctionalized nanocrystals was tested in immunoblot test, that confirmed preserved activity of attached molecules and lack of undesired nonspecific binding or precipitation on the assay membrane.
\end{abstract}

\section{Graphic abstract}

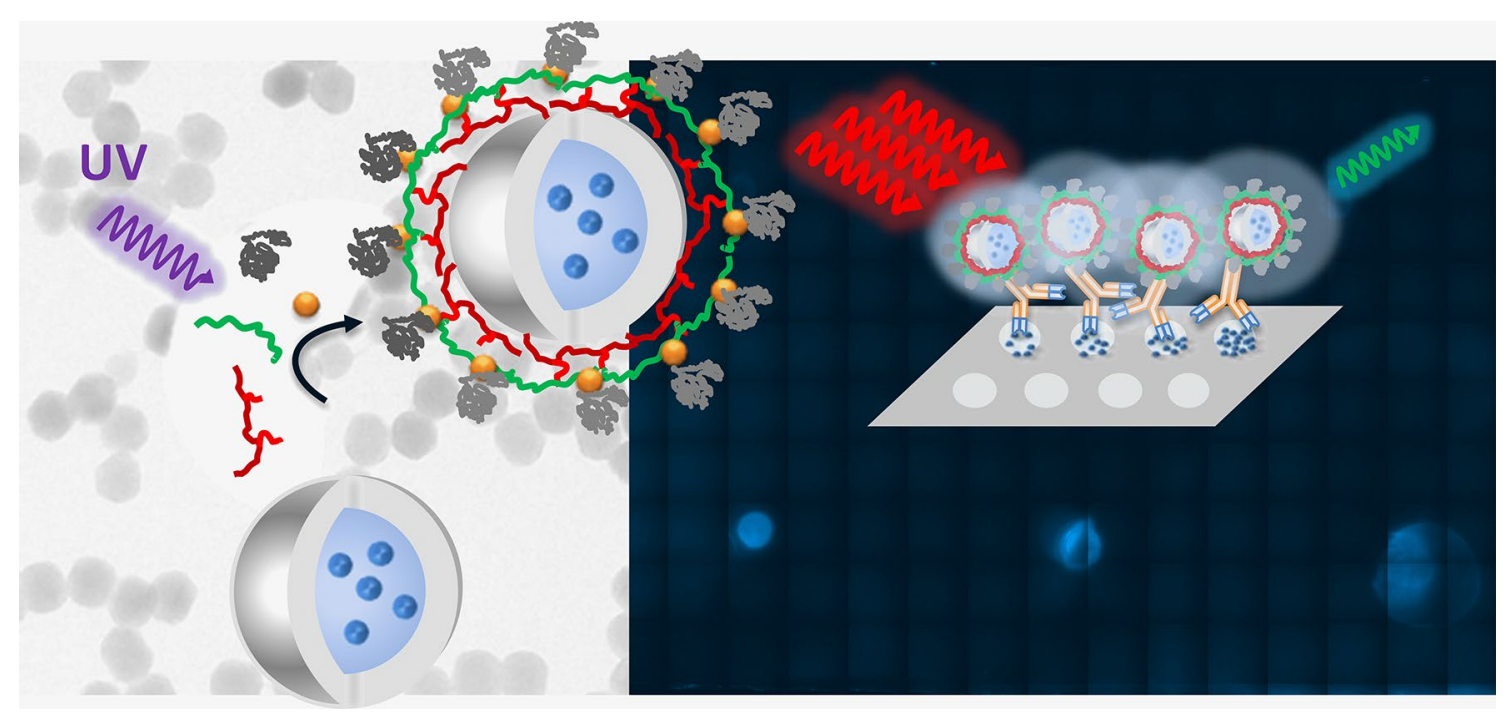

Extended author information available on the last page of the article 
Keywords Up-converting nanoparticles · Biofunctionalization $\cdot$ Photoactivatable linker $\cdot$ Dot blot $\cdot$ Protein detection

\section{Introduction}

Fluorescence-based detection and imaging are powerful tools for fundamental biology and practical diagnosis in medicine and therapy. In particular, fluorescence probes can be applied for studying molecular interactions and cellular systems including advanced diagnostics (e.g. analytes detection using southern and western blotting, hybridization microarrays, Real-Time PCR), bio-distribution analysis (e.g. distribution of drugs and drug carriers), imaging of live or fixed cells and tissues to describe activity, metabolism, morphology, and composition [1-4]. These techniques offer high sensitivity, multi-label possibility, relatively low cost, and wide range of commercially available bio-specific probes. Conventional fluorescent dyes, used as labels in bio-imaging and biological studies have some limitations and drawbacks. A particular disadvantage is an operation under visible or ultraviolet light excitation which leads to non-specific autofluorescence of the samples and thus decreased contrast of imaging that results in limited detection. The vast majority of organic dyes are sensitive to irradiation and the main issue in their utilization is photobleaching. Under excitation, many fluorescent organic molecules undergo irreversible chemical reactions after which they lose fluorescence capabilities [5, 6]. Although some chemical modifications of dyes were designed and radical scavengers are used, photobleaching is still one of the main limiting factor for most of the existing organic fluorophores to be applied in more demanding experiments like in-vitro tracking or time-lapse studies. A partial solution is provided by the development of near-infrared (NIR) fluorescent labels. In the NIR region, the observed background fluorescence is diminished and at the same time stability of organic fluorophores is increased. Furthermore, near-infrared light can penetrate tissues deeper than ultraviolet or visible radiation and causes less tissue damage than UV light. Therefore, non-destructive and deep imaging of living organisms in real-time became possible $[7,8]$. Nevertheless, only a slight difference between excitation and emission wavelengths together with still insufficient photostability of NIR dyes remains the challenge [8,9].

The mentioned drawbacks can be omitted by replacing standard NIR fluorophores with up-converting nanoparticles (UCNPs). Photon up-conversion is a process which results in anti-Stokes emission at wavelengths shorter (higher energy) than the excitation wavelength. UCNPs convert near-infrared light (usually 808 or $980 \mathrm{~nm}$ ) to visible and UV light and what is most important, they are not susceptible to photobleaching. Moreover, lanthanide-based nanocrystals offer narrowband and multicolor emission in UV/Vis/NIR range, large anti-Stokes shift, and long luminescence lifetimes (suitable for timeresolved background rejection). On the other hand, these materials are not suitable for biological applications in their native form $[10,11]$. One of the most widely used UCNPs-lanthanide-doped $\mathrm{NaYF}_{4}$ nanocrystals are usually obtained as oleate-capped hydrophobic nanoparticles dispersed in organic solvents. Owing to the character of capping molecules they are soluble solely in organic solvents and require additional treatment for transfer into the water environment $[12,13]$.

One may find numerous protocols for bio-functionalization of UCNPs, however, mainly due to poor efficiency of biofunctionalization, insufficient stability of functionalized nanoparticles in water or inability to form stable conjugates of nanoparticles with biomolecules, their application is still limited [14-26]. Despite these issues, more and more functionalization protocols allowed to demonstrate lanthanide-doped UCNPs as e.g. labels for human serum protein -Visceral adipose tissue-derived serpin detection [27]. Upconverting nanoparticle assemblies were recently also presented in excellent work as labels in multiplexed detection, monitoring, and in vivo imaging of small noncoding RNAs responsible for posttranscriptional regulation of gene expression [28]. UCNPs in the assembly were also used in many experiments for noninvasive temperature sensing, based on luminescence sensitivity to temperature changes [29].

Although many of the methods described in the literature lead to effective functionalization of nanomaterials surface, the issues with unspecific interactions may prevent successful utilization in some applications [30-33]. Due to specific requirements in the utilization of bio-functionalized UCNPs in dot blot assays, we focused to develop protocols allowing to obtain functionalized nanoparticles that lack nonspecific interactions with nitrocellulose and immobilon membranes.

A novel bio-conjugation technique based on water-soluble polymers derivatized with a photoactivatable linker was designed and characterized. The polymers with photoactivatable linker were applied in two-step protocol, providing a biocompatible coat effectively stabilizing nanocrystals and available for conjugation with bioactive molecules. In the next step, immunoglobulin-binding Protein $\mathrm{G}$ or goat antibodies were attached to coat UCNPs. The method allows obtaining functionalized nanocrystals that due to the lack of unspecific binding to dot blot membrane can be easily used for analyte detection. UCNPs-based dot blot reader was designed based on an inverted fluorescence microscope, which together with newly developed UCNPs functionalization technique, were characterized in terms of suitability for quantitative measurements of proteins. The new biofunctionalization technique was studied for $(\alpha)$ cubic and 
$(\beta)$ hexagonal phase $\mathrm{NaYF}_{4}$ that showed universality and potential for use with other matrices-based nanoparticles.

\section{Experimental}

\section{Materials and methods}

Nanocrystals were prepared using commercially available reagents without any further purification. Yttrium oxide $\mathrm{Y}_{2} \mathrm{O}_{3}(99.99 \%)$, ytterbium oxide $\mathrm{Yb}_{2} \mathrm{O}_{3}$ (99.99\%), erbium oxide $\mathrm{Er}_{2} \mathrm{O}_{3}$ (99.99\%), sodium trifluoroacetate (98\%), trifluoroacetic acid (ReagentPlus $\left.{ }^{\circledR}, 99 \%\right)$, Polyvinylpyrrolidone (PVP), $N$-(3-Dimethylaminopropyl)- $N$ '-ethylcarbodiimide hydrochloride EDC, N-Hydroxysuccinimide NHS, 1-octadecene (90\%) and ammonium fluoride $\mathrm{NH}_{4} \mathrm{~F}(\geq 98.0 \%)$ were purchased from ALDRICH Chemistry. Oleic acid (90\%) was purchased from ABCR GmbH \& Co. KG. Ethanol (96\% pure p.a.), methanol (pure p. a.), n-hexane (95\%) and acetone (pure p.a.) were purchased from Chempur (Poland). Chloroform (pure p.a.), sodium hydroxide $\mathrm{NaOH}$ (pure p.a.), acetic acid $(99.5 \%)$ were purchased from POCH S.A. (Poland), ANB-NOS ( $N$-5-azido-2-nitrobenzoyloxysuccinimide) was purchased from ThermoFisher Scientific. Carboxymethyldextran (CM-Dextran) was purchased from Sigma Aldrich. Sulfo-NHS-Diazirine, sulfosuccinimidyl 4,4'-azipentanoate was purchased from ThermoFisher. Anti-mouse antibodies isolated from goat (novex ${ }^{\mathrm{TM}}, \mathrm{H}+\mathrm{L}$; unconjugated, crossadsorbed) were purchased from Life Technologies.

To synthesize cubic and hexagonal $\mathrm{NaYF}_{4}: 20 \% \mathrm{Yb}^{3+}$ $2 \% \mathrm{Er}^{3+}$ nanocrystals, thermal decomposition of trifluoroacetate or acetate salts with some modifications has been used [34, 35]. Detailed synthesis protocols are described in Supporting Information. The structure, and morphology of nanoparticles were characterized by X-ray diffraction on X-ray diffractometer WAXS/SAXS - EMPYREAN (PANalytical), transmission electron microscope FEI Tecnai G2 X-TWIN and dynamic light scattering on Zetasizer Nano (Malvern ZS). The luminescent spectra of up-converting nanocrystals were measured with Andor Shamrock SR-303i spectrograph equipped with Newton EMCCD spectroscopy detector system.

\section{Synthesis and modifications of polymers}

Aminated PVP copolymer was synthesized according to work of P.I. Kitov [36] and was kindly provided by Dr. Pavel Kitov. $100 \mathrm{mg}$ of the aminated PVP copolymer was dissolved in $1.98 \mathrm{~mL}$ dimethyl sulfoxide (DMSO) followed by addition of $25 \mathrm{mg}$ ANB-NOS in $20 \mu \mathrm{L}$ DMSO. Reaction mixture was stirred overnight in the dark at room temperature. Subsequently $2 \mathrm{~mL}$ of Milli-Q water was added and non-bonded ANB-NOS was removed by dialysis. A small amount of final product was lyophilized for evaluation of concentration. Carboxylation of dextran was prepared following protocol described in Ref. [37]. Amination of carboxymethyl dextran was performed with EDC/NHS and ethylenediamine as amine group donor. Briefly, $500 \mathrm{mg}$ of Carboxymethyl-dextran (CM-Dextran) was dissolved in $3 \mathrm{~mL}$ of $0.1 \mathrm{M}$ MES buffer $\mathrm{pH} 4.8$, then $3.25 \mathrm{mmol}$ EDC$\mathrm{HCl}$ and NHS was added. The mixture was incubated $1 \mathrm{~h}$ with stirring at RT, followed by the addition of $15 \times$ molar excess of ethylenediamine titrated to $\mathrm{pH} 9.0$ by concentrated $\mathrm{HCl}$ (final $\mathrm{pH}$ of the reaction mixture should be $~ 8$ ). Amination was then provided for $2 \mathrm{~h}$. Finally, dextran was separated from other compounds by precipitation and washing with ethanol and acetone and dialysis to Milli-Q water. After dialysis it was lyophilized and the quantity of free amino groups was determined by 2,4,6-trinitrobenzene sulfonic acid (TNBS) assay.

\section{Bio-functionalization}

Bio-functionalization of the up-converting nanocrystals with the utilization of modified polymers can be divided into a couple of steps and is described below as well as in Fig. 1 in detail.

\section{Removing of organic ligand}

To attach functional groups the first step was to remove oleic acid. For this purpose $1 \mathrm{~mL}$ of UCNPs in chloroform $(60 \mathrm{mg}$ of nanocrystals $/ \mathrm{mL}$ ) was mixed with $1 \mathrm{~mL}$ of $1 \mathrm{M} \mathrm{HCl}$, then vortexed vigorously $5 \mathrm{~min}$ for protonation of carboxyl groups. An aqueous phase was collected and washed twice by centrifugation $(22,000 \mathrm{~g}, 20 \mathrm{~min})$ and redispersion in $\mathrm{mQ}$ water or dialyzed until the mixture reached $\mathrm{pH} \sim 3-4$.

\section{Initial coating}

$0.4434 \mathrm{mg}$ Polyvinylpyrrolidone conjugated with N-5-Azido-2-nitrobenzoyloxysuccinimide PVP-ANB-NOS ( $30 \mu \mathrm{L}, 14.78 \mathrm{mg} / \mathrm{mL}$ ) was added to the ligand-free UCNPs for overnight incubation. The free polymer was discarded with supernatant by centrifugation and functionalized UCNPs were dispersed in $1 \mathrm{~mL} \mathrm{mQ}$ water.

\section{Crosslinking with aminated dextran}

A solution of $30 \mathrm{mg}$ Dex- $\mathrm{NH}_{2}$ in $2 \mathrm{~mL} \mathrm{mQ}$ water was prepared followed by dropwise addition of $1 \mathrm{~mL}$ UCNP-PVP with continuous and intensive stirring. The vessel was placed on a magnetic stirrer and the sample was exposed to UV radiation $\left(1 \mathrm{~h} \lambda_{\max } 366 \mathrm{~nm}\right.$ ) generated by $4 \mathrm{~W}$ handlamp. For purification of double-coated UCNPs, gel column packed with a Sepharose CL-6B resin and equilibrated with 

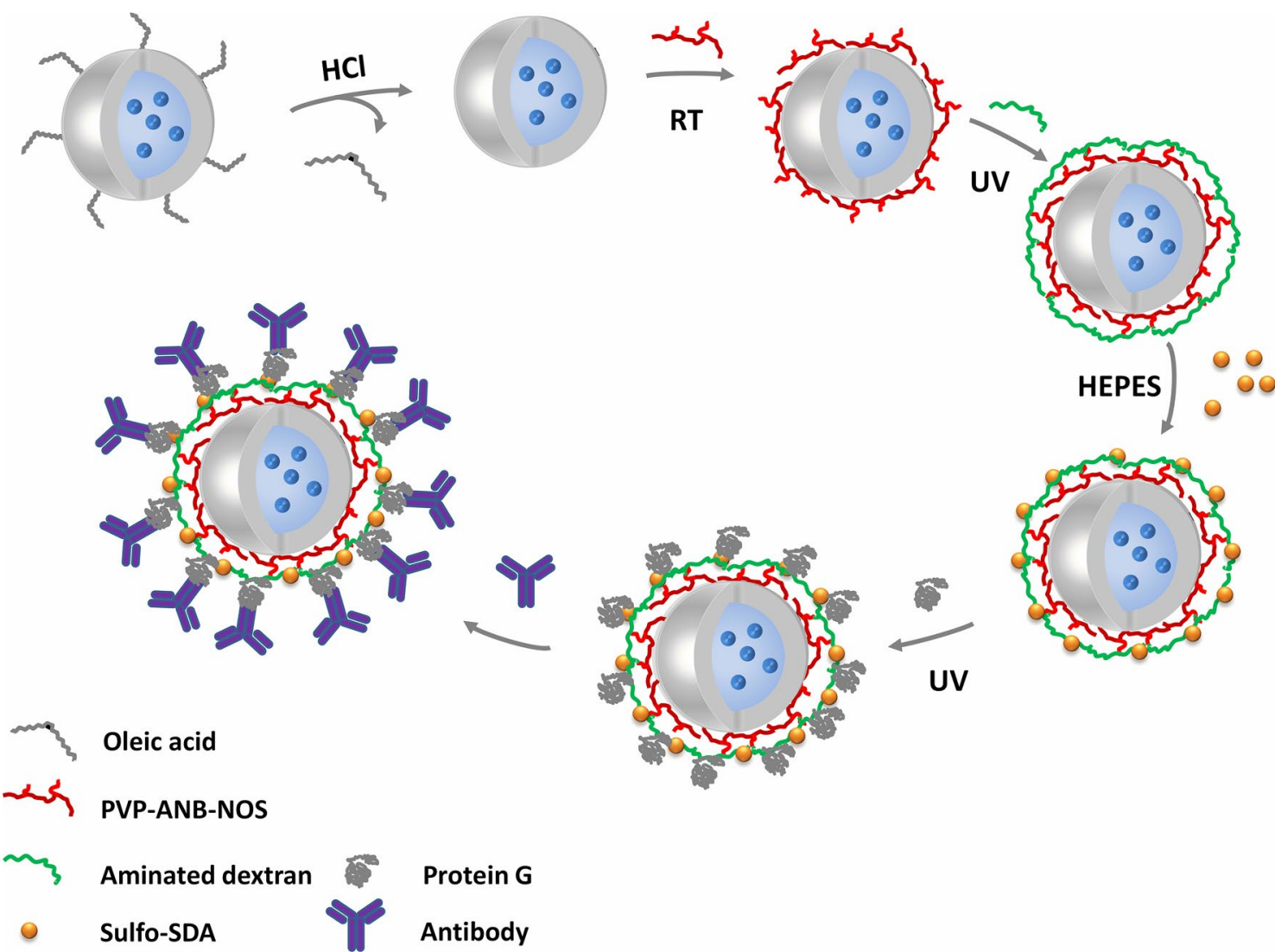

Fig. 1 General procedure of up-converting nanoparticles biofunctionalization. First step is removing oleate moieties by protonation with $\mathrm{HCl}$. Next step is incubation with PVP-ANB-NOS at room temperature (RT) followed by incubation with aminated dextran together with

$0.9 \% \mathrm{NaCl}$ (elution was monitored on refractometer) was used. In the end, NPs were centrifuged and redispersed in $3 \mathrm{~mL} 0.9 \% \mathrm{NaCl}$. The obtained nanoparticles dispersion was stable and precipitation was not observed.

\section{Conjugation}

$0.5 \mathrm{mg}$ sulfo-SDA (Sulfo-NHS-Diazirine, sulfosuccinimidyl 4,4'-azipentanoate) dissolved in $150 \mu \mathrm{L} \mathrm{mQ}$ water was added to $1 \mathrm{~mL}$ of UCNP-PVP-Dex- $\mathrm{NH}_{2}$ together with $50 \mu \mathrm{L} 0.5 \mathrm{M}$ HEPES buffer $\mathrm{pH}$ 8.14. After $1 \mathrm{~h}$ of incubation excess of the unbound linker was removed by dialysis to $\mathrm{mQ}$ water.

In the next step, $2.5 \mathrm{mg}$ Protein $\mathrm{G}$ (Pierce ${ }^{\mathrm{TM}}$ Recombinant Ig binding Protein $\mathrm{G}$ ) dissolved in $50 \mu \mathrm{L} \mathrm{mQ}$ water was added and the mixture was radiated again for $1 \mathrm{~h}$ by UV lamp. Excess of protein was removed by gel chromatography. Functionalized nanocrystals were then centrifuged and redispersed in $3 \mathrm{~mL}$ of $0.9 \% \mathrm{NaCl}$ solution for further use.

Alternatively, anti-mouse antibodies isolated from the goat were selected for conjugation. The procedure was not significantly altered except for additional step and the necessity of changing medium for antibodies to $0.9 \% \mathrm{NaCl}$.

ultraviolet (UV) treatment of the sample. The nanocrystals are then incubated with sulfo-SDA and HEPES buffer. The attachment of protein $\mathrm{G}$ is obtained by another exposure to UV light

\section{Dot-blot testing}

\section{Preparation}

Different types of antigens were used to examine the effectiveness of the developed protocol, including lipopolysaccharide (LPS) from Escherichia coli, mammalian antibodies or horse myoglobin. LPS was dissolved in $\mathrm{mQ}$ water using an ultrasonic bath and proteins were dissolved or diluted in $0.9 \% \mathrm{NaCl}$ to initial concentration $0.5 \mathrm{mg} / \mathrm{mL}$ or $0.2 \mathrm{mg} / \mathrm{mL}$. For experiments, series of solutions were prepared. $1 \mu \mathrm{L}$ of antigen solution was applied on a nitrocellulose membrane by pressing it very gently with the end of the pipette tip to damp the membrane without forcing a liquid flow with piston, instead letting capillary force to transfer sample onto the membrane (this method provides higher density of bound antigen which results in increased signal). Dried membranes were placed in $2 \mathrm{~mL}$ microcentrifuge tube with blocking solution ( $1.5 \mathrm{~mL} \mathrm{1 \%} \mathrm{BSA} \mathrm{in} \mathrm{TBS)} \mathrm{and} \mathrm{incubated}$ for $45-60 \mathrm{~min}$ at $37{ }^{\circ} \mathrm{C}$ with thermo-shaker. Afterwards, membranes were moved to another tubes with $2 \mathrm{~mL}$ of the appropriate solution of TBS containing: primary antibodies 
for "sandwich" type detection or UCNPs $(0.3 \mathrm{mg} / \mathrm{mL})$ for direct recognition as well as a secondary agent. Tubes with antibodies were set to a second incubation again at $37^{\circ} \mathrm{C}$ for the next $2.5 \mathrm{~h}$. Subsequently, blots were washed in $15 \mathrm{~mL}$ conical centrifuge tubes filled with $5 \mathrm{~mL}$ TBS-T $(0.05 \%$ Tween 20) and shaken 5 min twice with buffer changed one time. Membranes immersed in a solution containing UCNPs were incubated overnight and mixed gently on a rotator. Before reading they were washed as mentioned above (in certain cases second washing was prolonged to decrease background noise).

\section{Dot-blot reading and analysis}

Dot-blot reading was performed using an automated fluorescence microscope (AxioObserver.Z1 from Carl Zeiss) which was modified in-house to image in up-conversion mode. To obtain the image of single dot in the dot blot (diameter of $\sim 1 \mathrm{~mm}$ ), tiles feature in Zen Software (ZenBlue2011) was employed. All images were taken with objective 10xEC Plan-Neofluar and camera EMCCD Rolera EM-C ${ }^{2}$. The field of view for $10 \times$ objective is $1.4 \mathrm{~mm}$, which was further cropped down to accomplish non-even excitation beam profile. Therefore, to image single dot in a dot blot, $3 \times 3$ image mosaic were required. Typically, a whole dot blot strip was imaged and the whole mosaic image was then analyzed off-line. Special care was taken during the measurements to preserve excitation intensity and acquisition time to keep the experimental setup properties fixed to quantitatively compare the intensities of the dots. For the excitation of the up-converting nanoparticles laser diode $975 \mathrm{~nm}$ connected to a fluorescence microscope was used (Spectra Laser, Poland). A special filter cube was prepared with dichroic filter FF750-SDi01-25 and emission filter FF01-950/SP for the measurements. Further details regarding image acquisition, dot-blot reading, and analysis are provided in Supporting Information.

\section{Results and discussion}

Two types of up-converting core@ @hell nanocrystals were used for experiments, namely cubic $(\alpha)$ and hexagonal $(\beta)$ $\mathrm{NaYF}_{4}: \mathrm{Yb}^{3+} \mathrm{Er}^{3+} @ \mathrm{NaYF}_{4}$. The synthesized nanoparticles were dispersed in chloroform and stored at room temperature. $\alpha-\mathrm{NaYF}_{4}: \mathrm{Yb}^{3+} \mathrm{Er}^{3+} @ \mathrm{NaYF}_{4}$ emits orange/yellow light while $\beta-\mathrm{NaYF}_{4}: \mathrm{Yb}^{3+} \mathrm{Er}^{3+} @ \mathrm{NaYF}_{4}$ exhibits green luminescence under near-infrared excitation. The observed color under $975 \mathrm{~nm}$ laser excitation originates mainly from two emission bands (green ${ }^{4} \mathrm{~S}_{3 / 2} \rightarrow{ }^{4} \mathrm{I}_{15 / 2}$ and red ${ }^{4} \mathrm{~F}_{9 / 2} \rightarrow{ }^{4} \mathrm{I}_{15 / 2}$ ) relative intensity (Fig. 2).

The concept which assumes the use of polymers to modify the properties of nanoparticles is an inspiration for

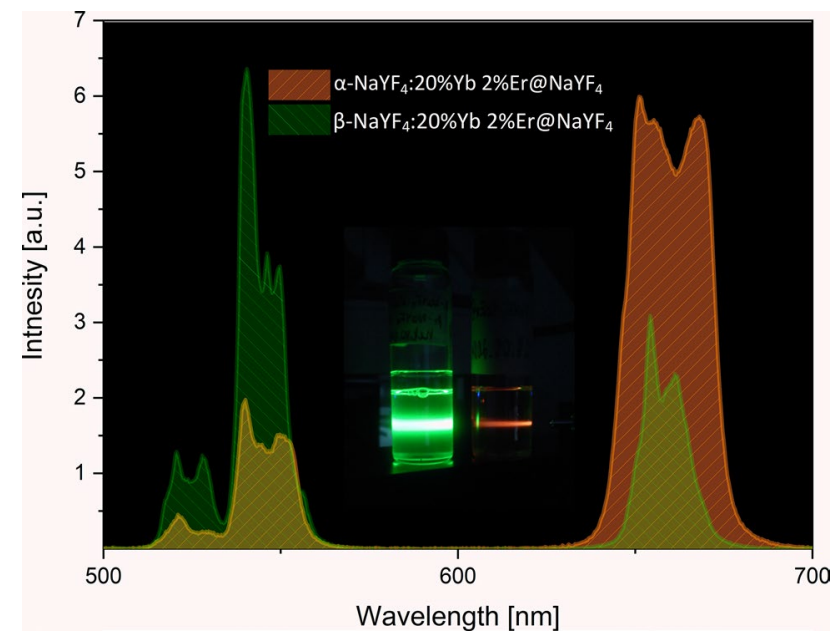

Fig. 2 Up-conversion luminescence spectra of $\alpha-\mathrm{NaYF}_{4}: \mathrm{Yb}^{3+} \mathrm{Er}^{3+} @$ $\mathrm{NaYF}_{4}$ and $\beta-\mathrm{NaYF}_{4}: \mathrm{Yb}^{3+} \mathrm{Er}^{3+} @ \mathrm{NaYF}_{4}$ nanocrystals under excitation with $975 \mathrm{~nm}$ laser diode. The acquisition time for spectra collection was $500 \mathrm{~ms}$ for cubic and $5 \mathrm{~ms}$ for hexagonal nanocrystals with the same power of excitation. The inset image shows up-conversion luminescence from colloidal solutions of nanocrystals- green from $\beta-\mathrm{NaYF}_{4}: \mathrm{Yb}^{3+} \mathrm{Er}^{3+} @ \mathrm{NaYF}_{4}$ and orange from $\alpha-\mathrm{NaYF}_{4}: \mathrm{Yb}^{3+} \mathrm{Er}^{3+} @$ $\mathrm{NaYF}_{4}$

researchers for at least several years [22, 38-41]. Polyvinylpyrrolidone is a common choice mostly due to its good biocompatibility, lack of toxicity, and solubility in many solvents including water. In nanotechnology PVP plays role as a structure and size regulating agent during the synthesis of different type of nanoparticles and also for their stabilization especially in an aqueous environment [41-43].

We used PVP-vinyl acetate copolymer to enable polymer functionalization with functional groups, e.g. amines. After preparation of aminated PVP, copolymer was ready for coupling to ANB-NOS containing amine reactive NHS ester. The linker provides photoactive nitrophenyl azide moiety for further coupling. It was used as a tool for coating and stabilization of bare UCNPs which also allowed for conjugation with proteins (BSA was chosen for a trial).

However, when we attempted functionalization using above procedure, we observed immediate precipitation of whole material while adding proteins to the solution of coated UCNPs.

To prevent precipitation and improve NPs stability, introduction of the second coating with dextran was necessary (Fig. 1). Dextran is a polysaccharide composed of glucose, characterized by good solubility, biocompatibility, lack of toxicity and with many documented applications in medicine [44-46]. Its carboxymethylated derivative had been aminated that allowed subsequent coupling to PVP located on UCNPs by photoactivation of ANB-NOS. Obtained nanoparticles were purified from an excess of dextran by size exclusion chromatography (Supplementary Information Fig. 
S3.). The stable dispersion of nanoparticles was obtained in acidic, neutral, and alkaline buffers. A few exceptions that we have found are buffers containing phosphates and carbonates. After contact even with very low concentrations of phosphates UCNPs precipitate almost immediately. After reaction Dextran still possess free amine groups ready for conjugation. Interestingly ANB-NOS cannot be used again as it causes precipitation of UCNPs. Due to that, another linker was employed, namely sulfo-SDA. It works in a similar way to ANB-NOS through NHS ester and UV-activated moiety, but it has a different structure and physicochemical properties. In the next step, UCNPs conjugates with antibodies or protein $\mathrm{G}$ were prepared.

To create a universal bioimaging tool NPs surface was coated with recombinant Protein G, a protein of bacterial origin capable of recognizing $\mathrm{Fc}$ fragments of mammalian antibodies, especially IgG type. Nanoparticles decorated with protein $\mathrm{G}$ can be easily coupled with immunoglobulins without engaging a chemical coupling. After photoconjugation excess proteins were removed by size exclusion chromatography. Purified nanoparticles were examined with Coomassie Protein Assay for the presence of protein and characterized via DLS measurements (Fig. S4 and Fig. S5). The measurements confirmed the monodispersity of the nanocrystals after functionalization. Zeta potential for nanoparticles after each step of functionalization was measured, however in the case of modified PVP-coated nanocrystals measurements were not possible due to precipitation of PVP-coated nanocrystals in $0.9 \% \mathrm{NaCl}$ solution. Zeta Potential which is associated with surface charge can be useful to predict particles' stability in the solution. Removing oleic acid by acid treatment results in a high positive charge of bare UCNPs $(36.8 \mathrm{mV})$. The addition of PVP and aminated dextran is reflected by the lower value of measured potential $(26.6 \mathrm{mV})$. A slightly negative value $(-3.1 \mathrm{mV})$ after conjugation with Protein $\mathrm{G}$ is consistent with a negative net charge of the protein. Despite the near-zero zeta potential of PVP-Dex-protein G coated nanoparticles, we did not observe large aggregates in DLS measurements.

A series of immunoassays with selected antigens served as a direct proof of protein $\mathrm{G}$ exposition on the nanoparticles surface. Conjugation of protein $\mathrm{G}$ to nanoparticles exerted no negative effect on the stability, size and shape of upconverters (Fig. 3). Solutions containing UCNPs functionalized with protein $\mathrm{G}$ were stored in a refrigerator without visible precipitation. Moreover, the activity of conjugate was preserved.

UCNPs functionalized with utilization of the developed protocol were tested in dot blot assays (Figs. 4 and 5). In a series of experiments optimal conditions for sensitive detection as well as the possibility to perform quantitative assays were verified.

Nitrocellulose transfer membrane (Bio-Rad) was chosen due to the good quality and simple application of the
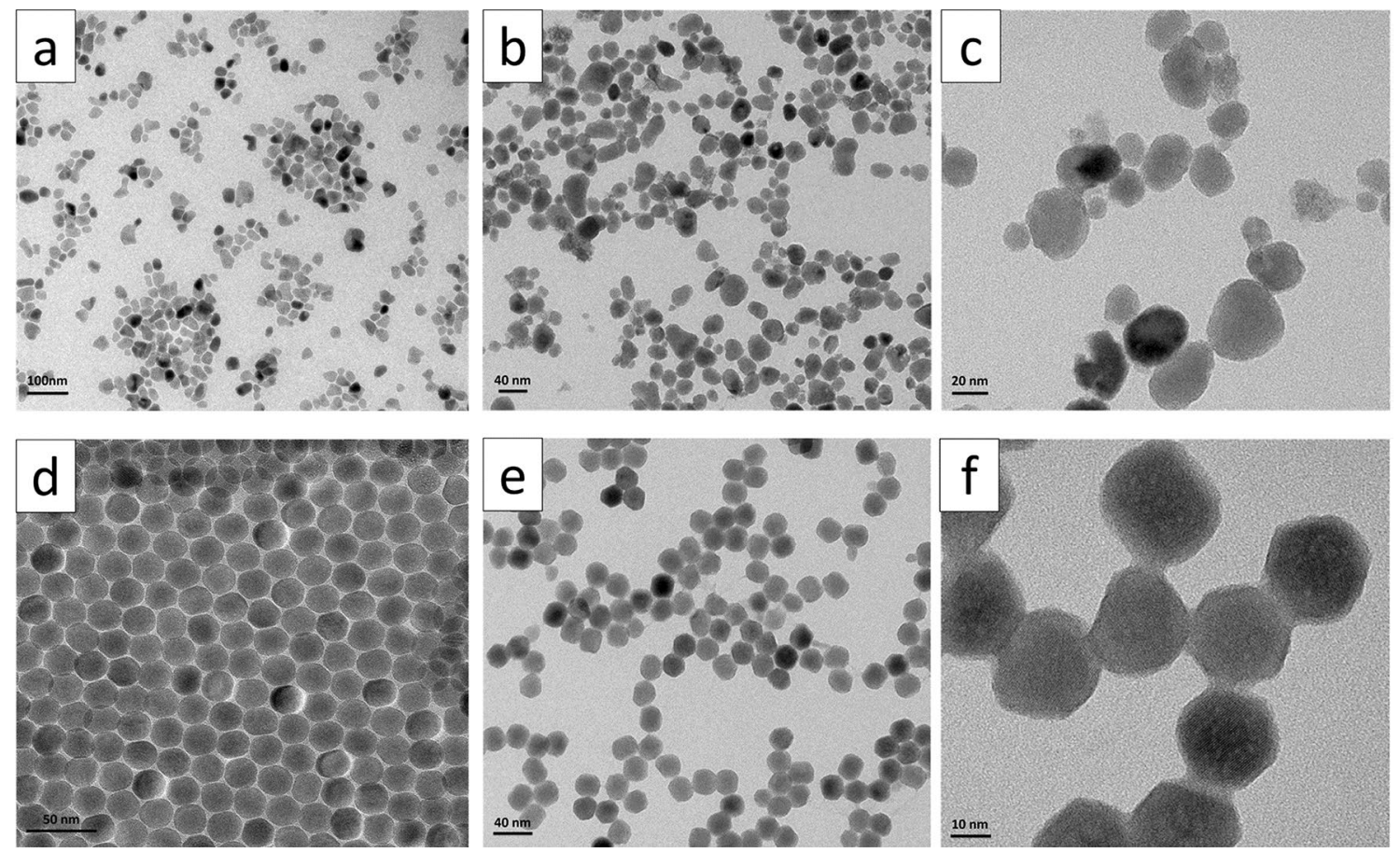

Fig. 3 TEM images of oleic acid capped (a, d) and biofunctionalized UCNPs (b, c, e, f). a-c Cubic phase nanocrystals; $\mathbf{d}-\mathbf{f}$ hexagonal phase nanocrystals 


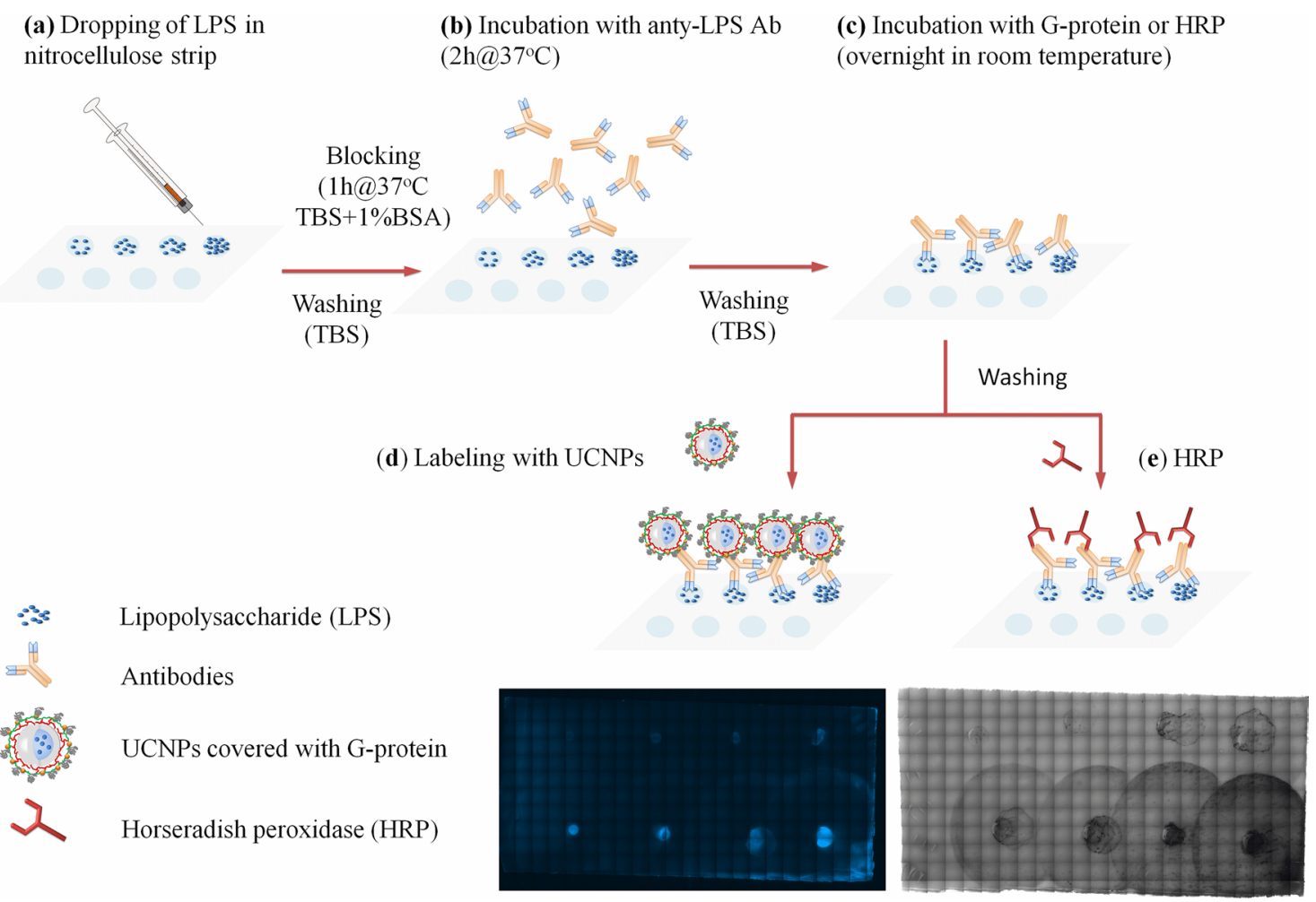

Fig. 4 Schematic presentation of dot blot experiments. Dot blot preparation included the attachment of lipopolysaccharide LPS to nitrocellulose strip (a), incubation with specific antibodies (b) followed by incubation with UCNPs covered with protein $\mathrm{G}(\mathbf{c}, \mathbf{d})$ or horserad-

samples. Tris-buffered saline is a common buffer in blotting and does not interact with coated UCNPs. The application method has a great impact on the limit of detection. Usually, $1 \mu \mathrm{L}$ of solution with dissolved antigen is put on the membrane as a drop hanging at the end of tip what results in a big dot with a broadly spread sample. The limited spreading can be obtained by a small modification where the tip with solution inside is touching membrane very gently until it slowly absorbed fluid only by capillary forces. This application method results in a significantly higher signal-to-background ratio. The utilization of functionalized nanocrystals in "sandwich" and direct type of dot blot assays is presented in Fig. 5a, b respectively. High background signal in blot in Fig. 5a is due to not thoroughly washed blot, however, it did not interfere with the collection of the signal, therefore this blot could be accepted. The up-conversion luminescence intensity is proportional to an analyte concentration that is shown in Fig. 5.

Initially, $\mathrm{NaYF}_{4}$ nanocrystals in $\alpha$-phase were examined. However, hexagonal ( $\beta$-phase) nanoparticles provided $\sim$ tenfold higher brightness (Fig. $5 \mathrm{c}, \mathrm{d}$ ). The upconversion luminescence intensity is proportional to the concentration of nanoparticles since these luminescent labels are barely susceptible to the chemical environment ish peroxidase HRP (c, e). The images represent dot blots in series of LPS dilutions, labelled with UCNPs. Images were taken under $975 \mathrm{~nm}$ diode excitation (left image) and bright field image of HRP labelled dot blot (right image)

and thus, after proper calibration UCNPs-based dot blots can be used for quantitative detection of antigen concentration. In the case of our initial studies, the detection limit for LPS with the utilization of two-step "sandwich" type dot blot and cubic UCNPs was about $2 \mu \mathrm{g} / \mathrm{mL}$ LPS taken from the dot in which the up-conversion luminescence signal could be measured (Fig. 5a). The detection limit for murine monoclonal antibodies with the utilization of hexagonal UCNPs in direct dot blot assay was $0.2 \mu \mathrm{g} / \mathrm{mL}$ (Fig. 5c) and was lower than mAb detection limit with the utilization of cubic UCNPs $(0.8 \mu \mathrm{g} / \mathrm{mL})$ (Fig. $5 \mathrm{~b}$ ). For human serum antibodies detection limit was $0.5 \mu \mathrm{g} / \mathrm{mL}$. However, some dots that were not recorded (luminescence signal was too low) can be still seen by the naked eye, so the detection limit can be lower. For precise quantitative analysis more optimization should be done and is not the aim of this work.

One of the important advantages of involving Protein $G$ is a fact that it is compatible with IgG type of antibodies originating from almost any organisms used in laboratory experiments, antibodies from i.e. mouse, rat, rabbit, goat, horse, human, sheep, cow, donkey and monkey. That gives functionalized up-converting nanoparticles functionality of a universal immunoimaging agent (Fig. 6). 

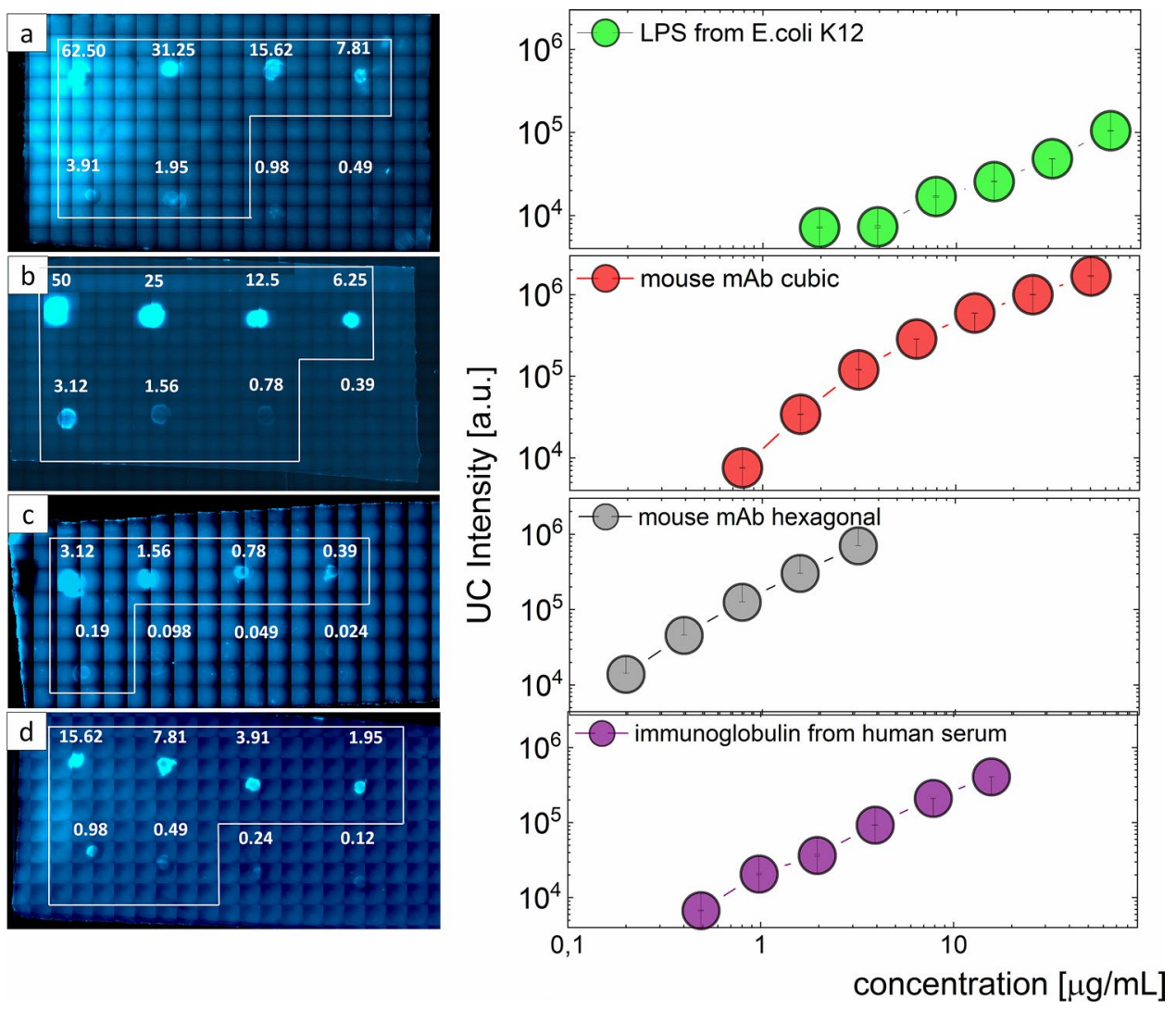

Fig. 5 Comparison of various dot blot assays. a Two-step ("sandwich") dot blot assay of LPS from E. coli K12 applied on nitrocellulose in series of dilutions then incubated with primary mouse antibodies $(5 \mu \mathrm{g} / \mathrm{mL}$ in TBS), subsequently stained with functionalized cubic $\mathrm{NaYF}_{4}: \mathrm{YbEr} @ \mathrm{NaYF}_{4}$ up-converting nanoparticles; dots correspond to $62.5-0.49 \mu \mathrm{g} / \mathrm{mL}$ LPS solutions applied on nitrocellulose membrane. b One-step (direct) dot blot assay of murine mAb (monoclonal antibodies) applied on nitrocellulose in a series of dilutions stained directly with functionalized up-converting cubic

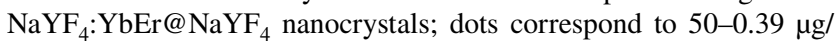

$\mathrm{mL}$ protein solutions applied on nitrocellulose membrane. c Murine $\mathrm{mAb}$ stained with hexagonal $\mathrm{NaYF}_{4}: \mathrm{YbEr} @ \mathrm{NaYF}_{4}$ nanoparticles; dots correspond to $3.125-0.024 \mu \mathrm{g} / \mathrm{mL}$ protein solutions. d Immunoglobulin from human serum stained with hexagonal up-converting nanoparticles; $15.62-0.12 \mu \mathrm{g} / \mathrm{mL}$ human serum antibodies solutions. In dot blot preparation $1 \mu \mathrm{L}$ of proper solution was used per single dot. The numbers above each dot correspond to concentration of the protein $(\mu \mathrm{g} / \mathrm{mL})$ used for particular dot. Right panel represents corresponding up-conversion intensity dependence on concentration of analyzed protein
Fig. 6 Versatility of UCNPsPVP-Dex-Protein G conjugate to antibodies derived from different mammalian species. a, b Mouse, two independent preparations; c goat; $\mathbf{d}$ rabbit; e human; f horse. $1 \mu \mathrm{L}$ of antibody solution was used per single dot. The membrane was stained with functionalized UCNPs and washed thoroughly to rinse out any particles that did not bind to the antibodies

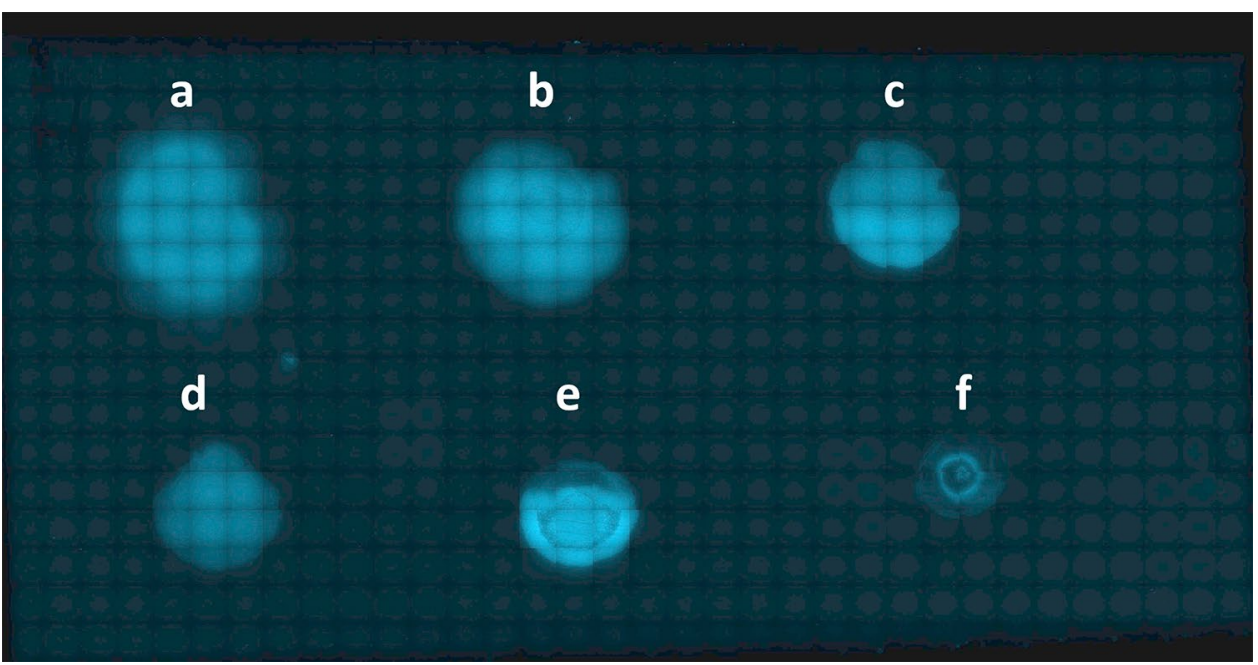




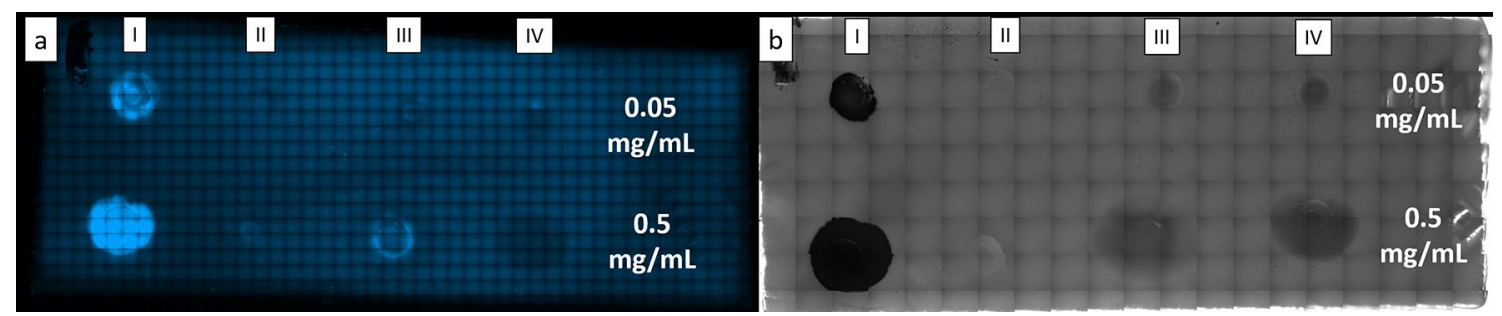

Fig. 7 Specificity assay for UCNPs functionalized with goat antimouse antibodies. a (I) mouse antibody, (II) BSA, (III) horse myoglobin, (IV) chicken egg white lysozyme. Upper row-concentration $0.05 \mathrm{mg} / \mathrm{mL}$; bottom row: $0.5 \mathrm{mg} / \mathrm{mL} ; 1 \mu \mathrm{L}$ of solution was used per

On the other hand, in some cases, high specificity of probes is required. Therefore, the developed functionalization method was used for direct pairing of antibodies with UCNPs, and the obtained probes were tested in specificity assays (Fig. 7a). A simultaneous test with goat antibodies horseradish peroxidase (Ab-HRP) was carried out to show that antibodies themselves, rather than nanoparticles, contribute to observed weak cross-reactions with other proteins (Fig. 7b). The properties of proteins used for biofunctionalization are crucial for the biological activity of the received conjugates. Because of that, the selection of high-quality antibodies is very important for successful experiments. Due to the antibodies' sensitivity to UV radiation, special care should be taken during the functionalization with UVsensitive linkers. The optimized UV irradiation conditions in the developed protocol are sufficient for effective photolinkers activation and gentle enough to sustain most proteins activity.

\section{Conclusions}

A new method for bio-functionalization of up-converting nanocrystals with the utilization of photo-linkers was proposed and described in detail. The modified UCNPs are suitable for utilization in dot blot assays since they lack unspecific binding to the nitrocellulose and immobilon membranes that was the main issue in the utilization of previously described functionalization methods. The conjugated protein activity was confirmed by the series of dot blot immunotests. The comparison between dot blot tests with utilization of cubic and hexagonal $\mathrm{NaYF}_{4}: \mathrm{Yb}^{3+} \mathrm{Er}^{3+}$ nanocrystals was also performed and showed significantly higher sensitivity in the case of hexagonal nanoparticles as compared to cubic nanoparticles. The developed functionalization method was shown to be applicable for building universal bioimaging tools as well as assays of high specificity.

Supplementary Information The online version contains supplementary material available at https://doi.org/10.1007/s40097-021-00404-X. single dot. b A simultaneous test with goat antibodies horseradish peroxidase Ab-HRP was carried out to show that antibodies themselves rather than nanoparticles contribute to observed weak crossreactions with other proteins

Acknowledgements Authors acknowledge financial support from Wrocław Research Centre EIT + within the project "The Application of Nanotechnology in Advanced Materials" - NanoMat (POIG.01.01.0202-002/08) financed by the European Regional Development Fund (Operational Programme Innovative Economy, 1.1.2).

\section{Declarations}

Conflict of interest The authors declare that they have no conflict of interest.

Open Access This article is licensed under a Creative Commons Attribution 4.0 International License, which permits use, sharing, adaptation, distribution and reproduction in any medium or format, as long as you give appropriate credit to the original author(s) and the source, provide a link to the Creative Commons licence, and indicate if changes were made. The images or other third party material in this article are included in the article's Creative Commons licence, unless indicated otherwise in a credit line to the material. If material is not included in the article's Creative Commons licence and your intended use is not permitted by statutory regulation or exceeds the permitted use, you will need to obtain permission directly from the copyright holder. To view a copy of this licence, visit http://creativecommons.org/licenses/by/4.0/.

\section{References}

1. Valeur, B., Berberan-Santos, M.N.: Molecular Fluorescence Principles and Applications. Wiley-VCH, Weinheim (2012)

2. Nishi, K., Isobe, S.I., Zhu, Y., Kiyama, R.: Fluorescence-based bioassays for the detection and evaluation of food materials. Sensors (Basel) 15, 25831 (2015)

3. Liu, Z., Zhou, X., Miao, Y., Hu, Y., Kwon, N., Wu, X., Yoon, J.: A Reversible fluorescent probe for real-time quantitative monitoring of cellular glutathione. Angew. Chemie Int. Ed. 56, 5812-5816 (2017)

4. Zhang, C., Xie, H., Zhan, T., Zhang, J., Chen, B., Qian, Z., Zhang, G., Zhang, W., Zhou, J.: A new mitochondrion targetable fluorescent probe for carbon monoxide-specific detection and live cell imaging. Chem. Commun. 55, 9444-9447 (2019)

5. Demchenko, A.P.: Photobleaching of organic fluorophores: quantitative characterization, mechanisms, protection. Methods Appl Fluoresc. 8(2), 022001 (2020)

6. Harper, I.S.: Fluorophores and their labeling procedures for monitoring various biological signals. In: Periasamy, A. (ed.) Methods 
in Cellular Imaging, pp. 20-39. Springer New York, New York (2001)

7. Hilderbrand, S.A., Weissleder, R.: Near-infrared fluorescence: application to in vivo molecular imaging. Curr. Opin. Chem. Biol. 14, 71-79 (2010)

8. Zhu, B., Sevick-Muraca, E.M.: A review of performance of nearinfrared fluorescence imaging devices used in clinical studies. Br. J. Radiol. 88, 20140547 (2015)

9. Jin, T., Huang, C., Cui, M., Yang, Y., Wang, Z., Zhu, W., Qian, $X .:$ Supramolecular ensembles modified by near-infrared dyes and their biological applications. J. Mater. Chem. B 8, 10686-10699 (2020)

10. DaCosta, M.V., Doughan, S., Han, Y., Krull, U.J.: Lanthanide upconversion nanoparticles and applications in bioassays and bioimaging: a review. Anal. Chim. Acta 832, 1 (2014)

11. Gnach, A., Bednarkiewicz, A.: Lanthanide-doped up-converting nanoparticles: merits and challenges. Nano Today 7, 532-563 (2012)

12. Prorok, K., Bednarkiewicz, A., Cichy, B., Gnach, A., Misiak, M., Sobczyk, M., Strek, W.: The impact of shell host $\left(\mathrm{NaYF}_{4} / \mathrm{CaF}_{2}\right)$ and shell deposition methods on the up-conversion enhancement in $\mathrm{Tb}^{3+}, \mathrm{Yb}^{3+}$ codoped colloidal $\alpha-\mathrm{NaYF}_{4}$ core-shell nanoparticles. Nanoscale 6, 1855-1864 (2014)

13. Chen, Y., D’Amario, C., Gee, A., Duong, H.T.T., Shimoni, O., Valenzuela, S.M.: Dispersion stability and biocompatibility of four ligand-exchanged $\mathrm{NaYF}_{4}$ : $\mathrm{Yb}$, Er upconversion nanoparticles. Acta Biomater. 102, 384-393 (2020)

14. Chen, Z., Chen, H., Hu, H., Yu, M., Li, F., Zhang, Q., Zhou, Z., Yi, T., Huang, C.: Versatile synthesis strategy for carboxylic acidfunctionalized upconverting nanophosphors as biological labels. J. Am. Chem. Soc. 130, 3023-3029 (2008)

15. Wang, F., Deng, R., Wang, J., Wang, Q., Han, Y., Zhu, H., Chen, X., Liu, X.: Tuning upconversion through energy migration in core-shell nanoparticles. Nat. Mater. 10, 968-973 (2011)

16. Chen, Q., Wang, X., Chen, F., Zhang, Q., Dong, B., Yang, H., Liu, G., Zhu, Y.: Functionalization of upconverted luminescent $\mathrm{NaYF}_{4}: \mathrm{Yb} / \mathrm{Er}$ nanocrystals by folic acid-chitosan conjugates for targeted lung cancer cell imaging. J. Mater. Chem. 21, 7661-7667 (2011)

17. Wong, H.T., Vetrone, F., Naccache, R., Chan, H.L.W., Hao, J., Capobianco, J.A.: Water dispersible ultra-small multifunctional $\mathrm{KGdF}_{4}: \mathrm{Tm}^{3}+, \mathrm{Y}^{\mathrm{b} 3}+$ nanoparticles with near-infrared to nearinfrared upconversion. J. Mater. Chem. 21, 16589-16596 (2011)

18. Mejías, R., Pérez-Yagüe, S., Gutiérrez, L., Cabrera, L.I., Spada, R., Acedo, P., Serna, C.J., Lázaro, F.J., Villanueva, Á., del Morales, M.P., Barber, D.F.: Dimercaptosuccinic acid-coated magnetite nanoparticles for magnetically guided in vivo delivery of interferon gamma for cancer immunotherapy. Biomaterials 32, 2938-2952 (2011)

19. Bogdan, N., Vetrone, F., Ozin, G.A., Capobianco, J.A.: Synthesis of ligand-free colloidally stable water dispersible brightly luminescent lanthanide-doped upconverting nanoparticles. Nano Lett. 11, 835-840 (2011)

20. Kumar, R., Nyk, M., Ohulchanskyy, T.Y., Flask, C.A., Prasad, P.N.: Combined optical and MR bloimaging using rare earth ion doped $\mathrm{NaYF}_{4}$ nanocrystals. Adv. Funct. Mater. 19, 853-859 (2009)

21. Zhang, Q., Song, K., Zhao, J., Kong, X., Sun, Y., Liu, X., Zhang, Y., Zeng, Q., Zhang, H.: Hexanedioic acid mediated surfaceligand-exchange process for transferring $\mathrm{NaYF}_{4}: \mathrm{Yb} / \mathrm{Er}$ (or $\mathrm{Yb} /$ $\mathrm{Tm})$ up-converting nanoparticles from hydrophobic to hydrophilic. J. Colloid Interface Sci. 336, 171-175 (2009)

22. Budijono, S.J., Shan, J., Yao, N., Miura, Y., Hoye, T., Austin, R.H., Ju, Y., Prud'Homme, R.K.: Synthesis of stable block-copolymer-protected NaYF 4: Yb 3+, Er 3+ up-converting phosphor nanoparticles. Chem. Mater. 22, 311-318 (2010)
23. Chen, G., Ohulchanskyy, T.Y., Law, W.C., Ågren, H., Prasad, P.N.: Monodisperse $\mathrm{NaYbF}_{4}: \mathrm{Tm}^{3+} / \mathrm{NaGdF}_{4}$ core/shell nanocrystals with near-infrared to near-infrared upconversion photoluminescence and magnetic resonance properties. Nanoscale $\mathbf{3}$, 2003-2008 (2011)

24. Olesiak-Banska, J., Nyk, M., Kaczmarek, D., Matczyszyn, K., Pawlik, K., Samoc, M.: Synthesis and optical properties of watersoluble fluoride nanophosphors co-doped with $\mathrm{Eu}^{3+}$ and $\mathrm{Tb}^{3+}$. Opt. Mater. (Amst) 33, 1419-1423 (2011)

25. Cao, T., Yang, T., Gao, Y., Yang, Y., Hu, H., Li, F.: Water-soluble $\mathrm{NaYF}_{4}: \mathrm{Yb} / \mathrm{Er}$ upconversion nanophosphors: Synthesis, characteristics and application in bioimaging. Inorg. Chem. Commun. 13, 392-394 (2010)

26. Johnson, N.J.J., Sangeetha, N.M., Boyer, J.C., Van Veggel, F.C.J.M.: Facile ligand-exchange with polyvinylpyrrolidone and subsequent silica coating of hydrophobic upconverting $\beta-\mathrm{NaYF}_{4}$ : $\mathrm{Yb}^{3}+/ \mathrm{E}^{\mathrm{r} 3}+$ nanoparticles. Nanoscale 2, 771-777 (2010)

27. Ali, M., Sajid, M., Khalid, M.A.U., Kim, S.W., Lim, J.H., Huh, D., Choi, K.H.: A fluorescent lateral flow biosensor for the quantitative detection of Vaspin using upconverting nanoparticles. Spectrochim. Acta Part A Mol. Biomol. Spectrosc. 226, 117610 (2020)

28. Qu, A., Sun, M., Xu, L., Hao, C., Wu, X., Xu, C., Kotov, N.A., Kuang, H.: Quantitative zeptomolar imaging of miRNA cancer markers with nanoparticle assemblies. Proc. Natl. Acad. Sci. U.S.A. 116, 3391-3400 (2019)

29. Sun, X., Sun, J., Dong, B., Huang, G., Zhang, L., Zhou, W., Lv, J., Zhang, X., Liu, M., Xu, L., Bai, X., Xu, W., Yang, Y., Song, $\mathrm{X}$., Song, H.: Noninvasive temperature monitoring for dual-modal tumor therapy based on lanthanide-doped up-conversion nanocomposites. Biomaterials 201, 42-52 (2019)

30. Sun, C., Li, H., Koidis, A., Chen, Q.: Quantifying Aflatoxin B1 in peanut oil using fabricating fluorescence probes based on upconversion nanoparticles. Spectrochim. Acta Part A Mol. Biomol. Spectrosc. 165, 120-126 (2016)

31. Salminen, T., Juntunen, E., Talha, S.M., Pettersson, K.: Highsensitivity lateral flow immunoassay with a fluorescent lanthanide nanoparticle label. J. Immunol. Methods. 465, 39-44 (2019)

32. Chen, Z., Zheng, W., Huang, P., Tu, D., Zhou, S., Huang, M., Chen, X.: Lanthanide-doped luminescent nano-bioprobes for the detection of tumor markers. Nanoscale 7, 4274-4290 (2015)

33. Xu, C.H., Ye, P.J., Zhou, Y.C., He, D.X., Wei, H., Yu, C.Y.: Cell membrane-camouflaged nanoparticles as drug carriers for cancer therapy. Acta Biomater. 105, 1-14 (2020)

34. Boyer, J.-C., Cuccia, L.A., Capobianco, J.A.: Synthesis of colloidal upconverting $\mathrm{NaYF}_{4}: \mathrm{Er}^{3+} / \mathrm{Yb}^{3+}$ and $\mathrm{Tm}^{3+} / \mathrm{Yb}^{3+}$ monodisperse nanocrystals. Nano Lett. 7, 847 (2007)

35. Abel, K.A., Boyer, J.C., Van Veggel, F.C.J.M.: Hard proof of the $\mathrm{NaYF}_{4} / \mathrm{NaGdF}_{4}$ nanocrystal core/shell structure. J. Am. Chem. Soc. 131, 14644-14645 (2009)

36. Kitov, P.I., Kotsuchibashi, Y., Paszkiewicz, E., Wilhelm, D., Narain, R., Bundle, D.R.: Poly(N-vinyl-2-pyrrolidone-co-vinyl alcohol), a versatile amphiphilic polymeric scaffold for multivalent probes. Org. Lett. 15, 5190-5193 (2013)

37. Hermanson, G.T.: Bioconjugate Techniques. Elsevier Inc., Amsterdam (2008)

38. Mashhadi Malekzadeh, A., Ramazani, A., Tabatabaei Rezaei, S.J., Niknejad, H.: Design and construction of multifunctional hyperbranched polymers coated magnetite nanoparticles for both targeting magnetic resonance imaging and cancer therapy. J. Colloid Interface Sci. 490, 64-73 (2017)

39. Liras, M., González-Béjar, M., Peinado, E., Francés-Soriano, L., Pérez-Prieto, J., Quijada-Garrido, I., García, O.: Thin amphiphilic polymer-capped upconversion nanoparticles: enhanced emission and thermoresponsive properties. Chem. Mater. 26, 4014-4022 (2014) 
40. Andresen, E., Resch-Genger, U., Schäferling, M.: Surface modifications for photon-upconversion-based energy-transfer nanoprobes. Langmuir 35, 5093-5113 (2019)

41. Jin, J., Gu, Y.J., Man, C.W.Y., Cheng, J., Xu, Z., Zhang, Y., Wang, H., Lee, V.H.Y., Cheng, S.H., Wong, W.T.: Polymer-coated $\mathrm{NaYF}_{4}: \mathrm{Yb}^{3+}, \mathrm{Er}^{3+}$ upconversion nanoparticles for charge-dependent cellular imaging. ACS Nano 5, 7838-7847 (2011)

42. Mirzaei, A., Janghorban, K., Hashemi, B., Bonyani, M., Leonardi, S.G., Neri, G.: Characterization and optical studies of PVPcapped silver nanoparticles. J. Nanostruct. Chem. 7, 37-46 (2017)

43. Song, Y.J., Wang, M., Zhang, X.Y., Wu, J.Y., Zhang, T.: Investigation on the role of the molecular weight of polyvinyl pyrrolidone in the shape control of high yield silver nanospheres and nanowires. Nanoscale Res. Lett. 9, 17 (2014)

44. Alibolandi, M., Mohammadi, M., Taghdisi, S.M., Abnous, K., Ramezani, M.: Synthesis and preparation of biodegradable hybrid dextran hydrogel incorporated with biodegradable curcumin nanomicelles for full thickness wound healing. Int. J. Pharm. 532, 466-477 (2017)

45. Basu, A., Kunduru, K.R., Abtew, E., Domb, A.J.: Polysaccharidebased conjugates for biomedical applications. Bioconjug. Chem. 26, 1396-1412 (2015)

46. Rees, K., Tran, M.V., Massey, M., Kim, H., Krause, K.D., Algar, W.R.: Dextran-functionalized semiconductor quantum dot bioconjugates for bioanalysis and imaging. Bioconjug. Chem. 31, 861-874 (2020)

Publisher's Note Springer Nature remains neutral with regard to jurisdictional claims in published maps and institutional affiliations.

\section{Authors and Affiliations}

\section{Małgorzata Misiak ${ }^{1,2}$ (D) Maciej Gawłowski ${ }^{1} \cdot$ Agnieszka Kowalczyk $^{1} \cdot$ Michał Skowicki $^{1} \cdot$ Katarzyna Prorok $^{2}$. Tomasz Lipiński ${ }^{1}$}

Małgorzata Misiak

m.misiak@intibs.pl

Tomasz Lipiński

Tomasz.Lipinski@port.lukasiewicz.gov.pl
1 Lukasiewicz Research Network-PORT Polish Center for Technology Development, Stabłowicka 147, 54-066 Wrocław, Poland

2 Institute of Low Temperature and Structure Research, Polish Academy of Sciences, Okólna 2, 50-422 Wrocław, Poland 